\title{
A multi-wavelength test of the FR I-BL Lac unifying model
}

\author{
E. Trussoni ${ }^{1}$, A. Capetti ${ }^{1}$, A. Celotti ${ }^{2}$, M. Chiaberge ${ }^{3,4}$, and L. Feretti ${ }^{4}$ \\ 1 Istituto Nazionale di Astrofisica (INAF) - Osservatorio Astronomico di Torino, Strada Osservatorio 20, \\ 10025 Pino Torinese (TO), Italy \\ 2 SISSA, via Beirut 2-4, 34014 Trieste, Italy \\ 3 Space Telescope Science Institute, 3700 S. Martin Drive, Baltimore, MD 21218, USA \\ 4 Istituto di Radioastronomia del C.N.R., via Gobetti 101, 40129 Bologna, Italy
}

Received 10 September 2002 / Accepted 13 March 2003

\begin{abstract}
We collect multi-wavelength measurements of the nuclear emission of 20 low luminosity FR I radio-galaxies to test the viability of the FR I-BL Lac unifying model. Although poorly sampled, the Spectral Energy Distributions (SED) of FR Is are consistent with the double peaked shape characteristic of BL Lacs. Furthermore while the distribution of the FR Is in the broad-band spectral index planes shows essentially no overlap with the regions where HBL and LBL are located, this can be simply due to the effects of relativistic beaming. More quantitatively, deriving the beaming Doppler factor of a given radiogalaxy from its X-ray luminosity ratio with respect to BL Lacs with similar extended radio luminosity, we find that i) the luminosity in all bands, ii) the value of the spectral indices, iii) the slope of the X-ray spectrum, iv) the overall SED shape, may be all simultaneously reproduced. However, the corresponding jet bulk Lorentz factors are significantly smaller than those derived for BL Lacs from other observational and theoretical considerations. This suggests to consider a simple variant of the unification scheme that allows for the presence of a velocity structure in the jet.
\end{abstract}

Key words. galaxies: active - galaxies: jets - galaxies: nuclei - BL Lacertae objects: general

\section{Introduction}

The unifying model for low power radio-loud Active Galactic Nuclei predicts that BL Lacs and Fanaroff-Riley I (FR I, Fanaroff \& Riley 1974) radio galaxies constitute the same class of objects, the difference being the orientation of their relativistic jets with respect to the line of sight. This scenario has been mainly supported (and tested) through the comparison of extended, presumably isotropic, properties of the two populations (see Urry \& Padovani 1995 for a review).

However it is also possible to verify and quantify the model parameters by estimating (or setting tight limits on) the nuclear emission in radio galaxies and directly compare it with the corresponding emission in BL Lacs. Recently the increased instrumental spatial and spectral resolution has in fact allowed the nuclear contribution in radio galaxies to be separated from the rest of the emission; there are now reliable nuclear flux estimates also in the X-ray and optical bands. Emission at optical and X-ray frequencies appears to well correlate with the radio core one, as shown by e.g. Canosa et al. (1999), Hardcastle \& Worrall (1999, hereinafter HW99) for the X-rays and Chiaberge et al. (1999, hereafter CCC99) for the optical case (as deduced from HST observations of FR I 3CR galaxies). These correlations suggest the same non-thermal origin

Send offprint requests to: E. Trussoni, e-mail: trussoni@to.astro.it of radiation in all three bands, thus implying little (if any) obscuration along the line of sight in FR Is. Furthermore, Capetti \& Celotti (1999, hereafter CC99) have been able to find for a subsample of (only) five FR Is that the optical core luminosity decreases by increasing the angle of the jet axis with respect to the line of sight, in agreement with the unifying scheme.

Following the same line of work, combining the information for individual sources in the different bands, Capetti et al. (2000, hereinafter Paper I) considered the spectral energy distributions of the same small sample analyzed in CC99, in the attempt to compare the whole individual SEDs of FR Is with those of BL Lac objects. In particular they considered the broad-band spectral indices $\left(\alpha_{\mathrm{ro}}, \alpha_{\mathrm{rX}}\right.$ and $\left.\alpha_{\mathrm{oX}}\right)$, which provide a good description of the SED, and also the X-ray spectral information, which provides a valuable tool to discriminate between the (steep) synchrotron and (flat) inverse Compton X-ray emission in BL Lacs. A similar broad-band approach has been later adopted also by Hardcastle \& Worrall (2000) and Bai \& Lee (2001). A general agreement with the unification model has been found through this comparison, with indications of the presence of two broad peaks, which characterize blazar spectra, also found in the FR I SEDs (with hints of the presence of High and Low frequency peak sources, as in BL Lacs, Giommi \& Padovani 1995). A very clear example of double peaked SED is that of Centaurus A (Chiaberge et al. 2001) in which both peaks can be accurately identified. However, it became 
Table 1. The sources and their core luminosities in the radio, $L_{\gamma \mathrm{r}}\left(\times 10^{23} \mathrm{~W} \mathrm{~Hz}^{-1}\right)$, and IR/optical/UV bands, $L_{v \mathrm{IR}}, L_{\gamma \mathrm{o}}$ and $L_{\gamma \mathrm{UV}}\left(\times 10^{19} \mathrm{~W} \mathrm{~Hz}{ }^{-1}\right)$.

\begin{tabular}{|c|c|c|c|c|c|c|c|}
\hline Source & Alt. name & $z$ & Envir. & $L_{v \mathrm{r}}^{a}$ & $L_{v I \mathrm{R}}$ & $L_{v \mathrm{o}}^{e}$ & $L_{\nu \mathrm{UV}}$ \\
\hline $3 C 29$ & & 0.0438 & A 119 & 3.5 & & 4.1 & $*$ \\
\hline $3 \mathrm{C} 31$ & NGC 383 & 0.0169 & Arp 331 & 0.51 & & 1.6 & \\
\hline $3 \mathrm{C} 66 \mathrm{~B}$ & & 0.0215 & & 1.6 & & 8.8 & $3.5^{h}, 2.5^{i}, 1.35^{m}$ \\
\hline $3 \mathrm{C} 78$ & NGC 1218 & 0.0288 & & 15 & & $96,85^{f}$ & $27.5^{m}$ \\
\hline $3 C 83.1$ & NGC 1265 & 0.0251 & Perseus & 0.26 & & 0.42 & \\
\hline $3 C \quad 189$ & NGC 2484 & 0.0413 & & 6.5 & & $25^{g}$ & \\
\hline $3 \mathrm{C} 272.1$ & M 84 & 0.0037 & Virgo & 0.047 & $1.0^{b}, 0.95^{c}, 1.1^{d}$ & $0.37^{g}, 0.13^{f}$ & \\
\hline $3 \mathrm{C} 277.3$ & & 0.0857 & Coma A & 1.8 & & $3.7,5.4^{f}$ & \\
\hline $3 \mathrm{C} 288$ & & 0.246 & & 39 & & 15.1 & \\
\hline $3 C 296$ & NGC 5532 & 0.0237 & & 0.84 & & 0.65 & \\
\hline $3 \mathrm{C} 310$ & & 0.0535 & & 4.5 & & 3.6 & \\
\hline $3 \mathrm{C} 317$ & & 0.0342 & A 2052 & 8.9 & $27^{b}$ & $5.1^{g}, 3.8^{f}$ & $0.22^{m}$ \\
\hline $3 \mathrm{C} 338$ & NGC 6166 & 0.0303 & A 2199 & 1.9 & $2.6^{b}$ & 3.0 & $0.14^{m}$ \\
\hline $3 \mathrm{C} 346$ & & 0.162 & & 120 & & 241 & $29^{l}$ \\
\hline $3 \mathrm{C} 348$ & & 0.154 & Her A & 4.9 & & 8.1 & \\
\hline $3 \mathrm{C} 442$ & NGC 7237 & 0.0262 & Arp 169 & 0.027 & & $0.23,0.13^{f}$ & \\
\hline $3 \mathrm{C} 449$ & & 0.0171 & & 0.21 & & 2.5 & * \\
\hline
\end{tabular}

${ }^{a} 5 \mathrm{GHz} ;{ }^{b} 16000 \AA{ }^{c} 11000 \AA{ }^{d} 20500 \AA{ }^{e} 7020 \AA{ }^{f} 5500 \AA{ }^{g} 8140 \AA{ }^{h} 4440 \AA ;{ }^{i} 3400 \AA{ }^{l} 1400 \AA{ }^{m}$; $2500 \AA$; * we did not use the HST data in the UV band for 3C 29 and 3C 449 since the nuclear emission of these sources is clearly affected by obscuration from extended (kpc-scale) dusty structures (Chiaberge et al. 2002).

clear that the simplest scenario involving a one velocity jet was not adequate to consistently reproduce the SEDs of BL Lacs and FR Is via beaming effects. In particular the emission in radio galaxies exceeded what was expected by de-beaming (at large angles) the typical BL Lac luminosity. It was therefore suggested the presence of a slower jet component (e.g. a layer of the jet) dominating the emission at larger angles (Chiaberge et al. 2000).

Given the relevance of such issues for both the unifying model itself and the physics of jets, we decided to further explore them by considering a significantly larger sample of sources. For this purpose we extend here the analysis of Paper I by constructing the SEDs for FR Is from the 3CR radio catalog for which multi-band observations are available and by comparing their SEDs with those of BL Lacs. The radio and optical data are those reported in CCC99. A few available infrared and UV observations not yet reported in the literature have been extracted from the HST archive. Most of the X-ray data have been taken from the recent literature, but again we also present here the analysis of Rosat pointed observations of a few objects which have not yet been presented elsewhere. From this selection we obtained a final sample of 16 radio galaxies, 20 including those examined in Paper I. From the study of this relatively large sample of objects we are able to deduce some further implications concerning the validity of the unified model.

In the following Sect. 2 we list the sources considered in this work and outline their main properties at different wavelengths, with particular emphasis on the X-ray observations. In Sect. 3 we build up the SED of our objects and we discuss their features and compare them with those of BL Lacs, while in Sect. 4 we test the BL Lac/FR I unification by considering the different beaming conditions. The main implications of our results on the validity of the unified scheme are summarized in the last Sect. 5. Throughout this work we adopt the following values for the cosmological parameters: $H_{0}=75 \mathrm{~km} \mathrm{~s}^{-1} \mathrm{Mpc}^{-1}$ and $q_{0}=0.5$. Spectral indices are defined by $F_{v} \propto v^{-\alpha}$.

\section{The sample and the multi-wavelength data}

The sources have been extracted from the $3 \mathrm{CR}$ for which X-ray data are available and core emission has been detected in the HST images (CCC99). In this list we have not considered M 87, that will be discussed separately, and 3C 84, that appears to be a very peculiar object (sometimes it is classified as a BL Lac) and highly variable at all frequencies. Conversely we have kept in the sample 3C 346, that in most publications is classified as a narrow line, FR II radio galaxy. We remark also that 3C 348 (Hercules A) has properties similar to both FR I and FR II radio galaxies and its total radio luminosity is more typical of FR IIs than FR Is. We have also considered in our sample 3C 189 , which is part of the B2 sample of radio galaxies recently studied using HST data by Capetti et al. (2001), although this source is not included in the revised version of the $3 \mathrm{C}$ cat$\operatorname{alog}(3 \mathrm{CR})$.

In the following we outline the main properties of our targets, discussing separately the data at radio and IR/optical/UV frequencies (Table 1) and those at X-ray energies (Table 2).

Radio observations. For the radio core luminosities we referred to the VLA observations at $5 \mathrm{GHz}$ of Giovannini et al. (1988) and Morganti et al. (1993), reported in CCC99. For some sources VLBI data also are available, however we prefer to use the lower resolution VLA observations as they have been obtained on scales similar to the measurements at the other wavelengths. The flux variability of the radio core is generally negligible for our sources. Fluctuations of $\approx 20 \%$ have been found in 3C 66B (Hardcastle et al. 1996), whereas in 3C 338, 
the most strongly variable radio galaxy known of our sample, the core flux may vary up to $\approx 60-70 \%$ (Giovannini et al. 1998). Optical, infrared and UV observations. Most of the HST observations in CCC99 were performed with the WFPC2 using the filter F702W; for a few sources data with different filters are also available. In the public archive at STSCI observations with the HST/NICMOS are found at infrared frequencies for 3C 272.1 with the broad-band filters F110W, F160W and F205W, and for 3C 317 and 3C 338 with F160W. The analysis of these data have been performed as in Paper I. UV images of 3C 66B and 3C 346 have been taken with the HST/FOC (at $\lambda=4440 \AA$ and $3440 \AA$ ) and HST/STIS (at $\lambda=1400 \AA$ ), respectively. All these observations have been performed in the years 1994-1996. So far repeated HST observations are available only for a few sources. In particular, 3C 66B shows a decrease in flux by a factor $\approx 2$ in the optical between 1995 and 2000, while 3C 317 has revealed strong variability in the UV (by a factor 10) between 1994 and 1999 (Chiaberge et al. 2002). Other UV data of 3C 66B, 3C 78, 3C 317 and 3C 338 are taken from Chiaberge et al. (2002).

All the fluxes have been corrected for the galactic extinction, but some amount of local absorption may be present. In Paper I we estimated that, based on the small dispersion of the radio/optical correlation for FR I nuclei, such local extinction does not exceed 2 mag in the optical. This result is confirmed by recent UV observations (Chiaberge et al. 2002), which implies an underestimate of the luminosity of by factor $\lesssim 6$ (this is probably the case in $3 \mathrm{C} 272.1$ where a central dust lane is present, CCC99). However, this is not critically relevant for the construction of the SED (for details see Paper I).

$X$-ray observations. We discuss in more detail the observations in the X-ray band that are particularly crucial for us to be able to define the structure of the SED.

We must point out that in instruments with poor imaging capability the nuclear X-ray flux may be contaminated by spurious sources, like X-ray binaries, jets, etc. However the correlation between the radio and X-ray core luminosities (Canosa et al. 1999, HW99), suggests that in general the jet emission should provide the most relevant contribution to the nuclear flux. We remind in particular that for the radio galaxies of our sample the integrated contribution from binaries to the total emission is not relevant (see e.g. Fabbiano 1989). Furthermore from Chandra data it has been deduced that emission from $\mathrm{X}$-ray jets is not the dominant source of high energy photons in these radio galaxies (Hardcastle et al. 2001; Worral et al. 2001). It has also been proposed that advection dominated disks (ADAF) could contribute to the core emission from radio to hard X-ray energies (see e.g. Narayan \& Yi 1995; Di Matteo et al. 2000, 2001). In normal elliptical galaxies it has been excluded a relevant contribution from the ADAF to the nuclear luminosity (Loewenstein et al. 2001). Chandra observations suggest that this should be also the case for radio galaxies (Di Matteo et al. 2002; Pellegrini et al. 2003). The above considerations leave us with extended hot galactic gas as the main source of contamination.

The data on our sources are quite heterogeneous, as they have been collected from different instruments and satellites. In all cases the luminosities have been re-scaled to the soft X-ray
Table 2. Data on X-ray nuclear emission used for the SED (see the Appendix for details).

\begin{tabular}{cccc}
\hline \hline Source & $L_{v \mathrm{X}}^{a}$ & $\alpha_{\mathrm{X}}$ & Instrum. (refer.) \\
\hline 3C 29 & $5.5^{\star}$ & 1.0 & PSPC (1) \\
3C 31 & 0.46 & $0.5_{-0.2}^{+0.3}$ & Chandra (2) \\
3C 66B & $9.5_{-0.9}^{+0.2}$ & $0.3_{-0.4}^{+0.3}$ & PSPC (1) \\
3C 78 & $32_{-17}^{+10}$ & 1.0 & SAX (3) \\
3C 83.1 & $3.6^{\star}$ & 1.0 & PSPC (4) \\
3C 189 & 14 & $1.1_{-0.4}^{+0.1}$ & Chandra (5) \\
3C 272.1 & $0.051_{-0.003}^{+0.003}$ & $1.3_{-0.1}^{+0.1}$ & Chandra (6) \\
3C 277.3 & $<5.3$ & 1.0 & PSPC (1) \\
3C 288 & $<2100$ & 1.0 & PSPC (4) \\
3C 296 & $6.0^{\star}$ & 1.0 & HRI (4) \\
3C 310 & $16^{\star}$ & 1.0 & HRI (4) \\
3C 317 & $30_{-23}^{+21}$ & $0.1_{-0.7}^{+0.3}$ & PSPC (1) \\
3C 338 & $0.53_{-0.11}^{+0.11}$ & $0.5_{-0.4}^{+0.5}$ & Chandra (7) \\
3C 346 & $618_{-160}^{+110}$ & $0.7_{-0.2}^{+0.2}$ & ASCA (8) \\
3C 348 & 234 & 1.0 & PSPC, ASCA, SAX (9) \\
3C 442 & $<1.9$ & 1.0 & HRI (4) \\
3C 449 & $0.89^{\star}$ & 1.0 & PSPC (4) \\
\hline
\end{tabular}

${ }^{a} \times \overline{10^{16} \mathrm{~W} \mathrm{~Hz}^{-1} \text { (at } 1 \mathrm{keV} \text { ). For each object } N_{\mathrm{H}} \text { is fixed to its galactic }}$ value, except for 3C 272.1 where it is deduced from the fit $\left(N_{\mathrm{H}}=\right.$ $\left.2.7_{-0.3}^{+0.3} \times 10^{21} \mathrm{~cm}^{-2}\right)$. In the sources marked with ${ }^{\star}$ contamination from thermal emission may be present.

References: (1) this work; (2) Hardcastle et al. (2002); (3) Trussoni et al. (1999); (4) HW99; (5) Worral et al. (2001); (6) Finoguenov \& Jones (2001); (7) Di Matteo et al. (2000); (8) Worrall \& Birkinshaw (2001); (9) Siebert et al. (1999), Trussoni et al. (2001).

energy range of Rosat (0.1-2.4 keV). Most of the data reported here have been extracted from recent publications, while we directly analyzed the archival Rosat data of $3 \mathrm{C} 29,3 \mathrm{C} 66 \mathrm{~B}$, 3C 189, 3C 277.3 and 3C 317 not yet discussed in the literature.

Very recent Chandra data have been collected for $3 \mathrm{C} 31^{1}$ (Hardcastle et al. 2002), 3C 66B (Hardcastle et al. 2001), 3C 189 (Worral et al. 2001), 3C 272.1 (Finoguenov \& Jones 2001), 3C 317 (Blanton et al. 2003) and 3C 338 (Di Matteo et al. 2000). In all sources a point-like core has been detected, with flux almost unabsorbed locally $\left(N_{\mathrm{H}} \lesssim\right.$ few units of $10^{21} \mathrm{~cm}^{-2}$ ), however only for 3C 31, 3C 66B and 3C 272.1 is there a good evaluation of the spectral slope.

For six objects the core emission has been searched for through spectral fits with two-component models: thermal (from the environment) + power law (from the nucleus). A positive detection has been obtained for 3C 78 (BeppoSAX, Trussoni et al. 1999), 3C 346 (ASCA and Rosat, Worral \& Birkinshaw 2000) and 3C 348 (BeppoSAX, Rosat and ASCA, Siebert et al. 1999; Trussoni et al. 2001), although with poor, if any, estimate of the spectral index. From our spectral analysis of the Rosat/PSPC observations of 3C 29, 3C 277.3 and 3C 317 only an upper limit to the core emission has been deduced for

${ }^{1}$ We reconsider here this source that was previously analyzed in Paper I using Rosat data. 
the first two radio galaxies while for $3 \mathrm{C} 317$ non-thermal flux has been detected with very flat spectrum $\left(\alpha_{\mathrm{X}} \approx 0.1\right)$. For all those sources with no estimate of the spectral index, the X-ray luminosities have been deduced assuming $\alpha_{\mathrm{X}}=1$.

Finally, for the remaining six radio galaxies (3C 83.1, 3C 288, 3C 296, 3C 310 and 3C 449) we referred to the results of HW99 who, exploiting the imaging capabilities of the HRI and PSPC of Rosat, looked for the presence of point-like components coinciding with the active nuclei. As a small thermal region may be present in the central regions of radio galaxies the nuclear flux can be overestimated, mainly for distant sources (as e.g. in 3C 465, at the center of cluster A 2634, see Paper I). However the correlation between the radio and X-ray core luminosities suggests that in most of the detected cores the non-thermal component cannot be a negligible fraction of the total emission. In any case the values of the luminosities will be considered as upper limits of their nuclear emission (marked with a star in Table 2).

For $\approx 2 / 3$ of the targets repeated X-ray observations are available. However in most cases the count rate is too low (or only upper limits exist) to obtain information on the possible presence of variability. From ASCA and Rosat data a slight decrease of the emission has been found in 3 C 346 over $\approx 1.5 \mathrm{yr}$. In contrast, from two PSPC and one Chandra observation it turns out that the X-ray core flux from $3 \mathrm{C}$ 66B has decreased by a factor $\approx 4$ in 9 years. A similar trend has been found for $3 \mathrm{C} 317$, although the large statistical errors do not allow a definite conclusion. Out of the repeated observations of these three targets we use those closer in time to the HST pointings.

More details on the observations and the X-ray properties of the other objects not discussed in HW99 are reported in the Appendix.

\section{Results}

As already mentioned in the Introduction, the correlations found between the luminosities of the nuclei of FR Is in different spectral bands, from radio to X-ray energies, suggest a common non-thermal origin for the emission form these nuclei, occurring in the inner parts of the relativistic jets. Our aim is to use the data to compare the multi-wavelength properties of the nuclear emission of FR Is with those of BL Lacs, in order to derive information on the jet properties. In particular we examine the Spectral Energy Distribution, the intensity of the X-ray emission and the values of the broad-band spectral indices.

\subsection{The Spectral Energy Distribution}

The main characteristic of BL Lac SEDs is that they can be well described as composed by two broad peaks. The lower energy one, most likely due to synchrotron emission, is located in the mm-IR or UV-soft/X-ray bands in Low and High peak BL Lacs, respectively (HBL and LBL, in the terminology introduced by Giommi \& Padovani 1995). The higher energy component comprises the X-ray and $\gamma$-ray range and is possibly due to inverse Compton emission. Therefore, it is important to look for the presence of such two components also in the SEDs of FR Is, to investigate if indeed their nuclear emission is dominated by non-thermal radiation, only de-beamed with respect to BL Lacs. Positive indications of the compatibility of the behaviour of such a spectral shape with the (although poorly sampled) SEDs of FR Is have been found in Paper I. Here we further investigate the SED shape for a larger number of sources, and with better sampled energy distributions, and try to locate the positions of the peak of the synchrotron component.

The SEDs of our objects are shown in Fig. 1. All of them present an increase of the emission from radio up to $\approx$ infrared wavelengths, while significant differences can be seen at higher frequencies. Indications of the shape of the SEDs and on the location of the emission peak can be inferred from the relative intensity of the optical and X-ray emission and - as already mentioned - from the X-ray spectral index. Unfortunately, for half of the sources we have only an upper limit to the nuclear non-thermal X-ray emission (see Table 2). We do not further consider here the sources for which the $\mathrm{X}$-ray limit is not significant (3C 83.1, 3C 288, 3C 296, 3C 310 and 3C 442), whereas in three cases (3C 29, 3C 277.3 and 3C 449) the limit is still useful to provide constraints on the SED.

In 3C 78, 3C 272.1 and 3C 449, the X-ray luminosity is significantly lower than that of the optical component. This suggests the existence of a minimum approximately around $\mathrm{X}$-ray energies, in the region of transition from synchrotron to Compton emission and it is supported by the steep X-ray spectrum of $3 \mathrm{C} 272.1$.

In five radio galaxies $(3 \mathrm{C} 31,3 \mathrm{C} 66 \mathrm{~B}, 3 \mathrm{C} 317,3 \mathrm{C} 338$ and $3 \mathrm{C} 346$ ) the X-ray spectrum is rather flat (the SED is rising at these energies) suggesting that the synchrotron peak must be located at lower frequencies. Even though the overall shape of the SED may be affected by spurious emission in the IR/optical band (e.g. by dust emission) the above interpretation seems to be confirmed by the comparison of the SED slope in the IR/optical/UV spectral region. In fact in all these cases the slope is decreasing, suggesting for the presence of a minimum and for a location of the synchrotron peak at $\sim$ IR frequencies, or lower. The SEDs of these sources are then qualitatively similar to the SEDs of LBL: the X-ray emission is plausibly of Compton origin with a flat spectrum. Very similar properties are present in the SED of 3C 270 (see Paper I).

Conversely, the data for 3C 29, 3C 189, 3C 277.3 and 3C 348 (similar to 3C 465 discussed in Paper I) do not allow to characterize the shape of their SEDs.

While these findings generally support the presence of a double peaked SED of FR Is, it is necessary to stress that the luminosity scatter of these objects ranges over $\sim 2-3$ order of magnitudes in the radio and X-ray bands, and it is impossible to derive any trend with the source power.

\subsection{Comparison between the $X$-ray emission in FR Is and BL Lacs}

Measurements of the nuclear emission in FR I radio galaxies and their comparison with the luminosities of BL Lacs provide us with a direct measure of the amount of beaming affecting the two different classes of objects. For this test we must consider 

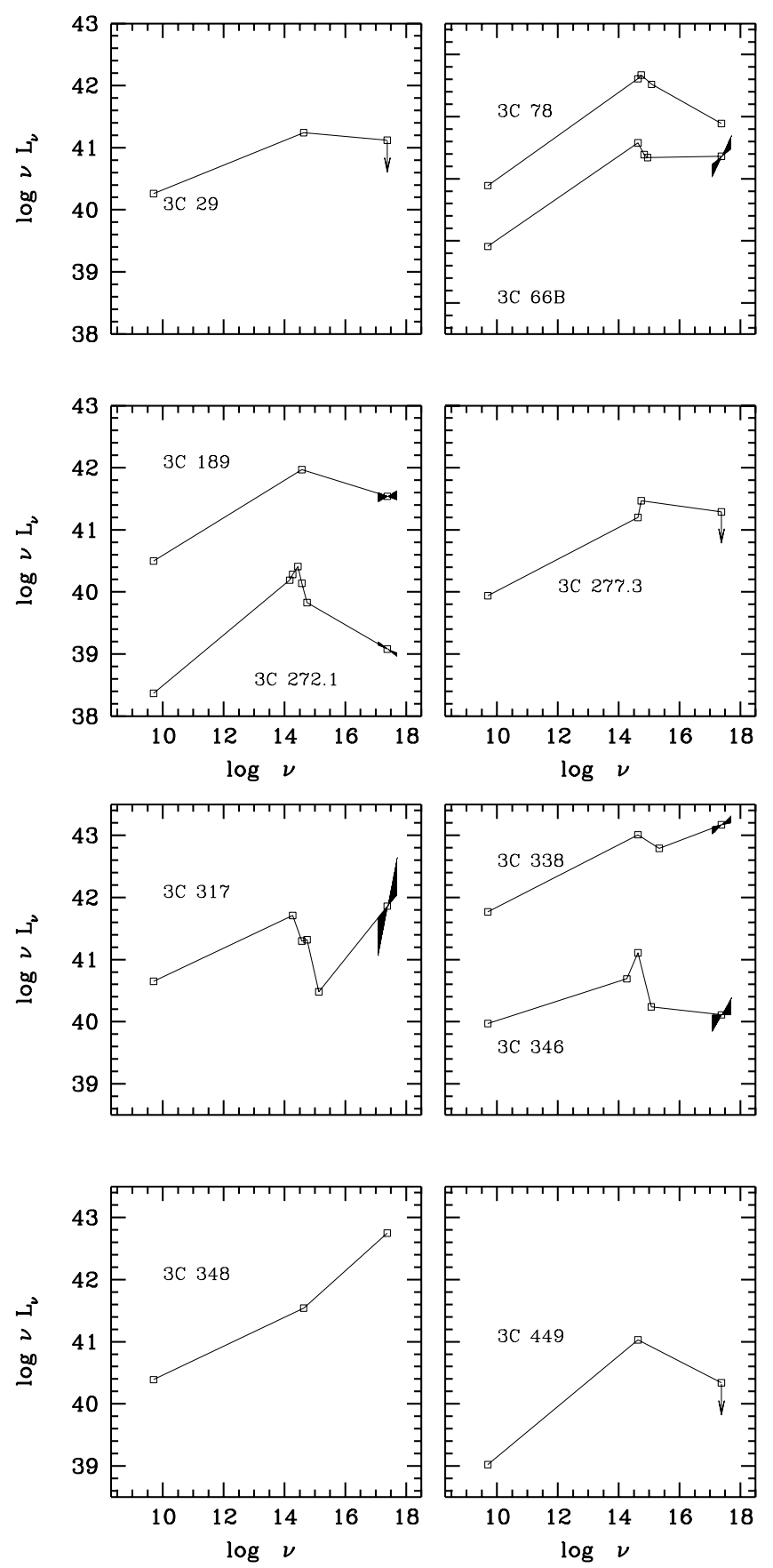

Fig. 1. Spectral Energy Distribution of radio galaxies. The values of $v L_{v}$ are in $\operatorname{erg~s}^{-1}$.

objects of similar large scale properties, which do not depend on orientation. In particular, they must share a common range of extended radio luminosity as this is linked to the energy carried by the relativistic jet, which is plausibly at the origin of the nuclear emission.

The same method has been applied both in the optical and radio bands to the $3 \mathrm{C}$ and the $\mathrm{B} 2$ samples of FR I radio galaxies (Chiaberge et al. 2000; Capetti et al. 2001). The luminosity ratio between FR Is and BL Lacs was found to be $L_{\mathrm{BL}} / L_{\mathrm{FR} \mathrm{I}} \approx$ $10^{2.5}$ and $L_{\mathrm{BL}} / L_{\mathrm{FR} \mathrm{I}} \approx 10^{3.9}$ in the radio and optical bands, respectively. These authors deduced that the difference in nuclear luminosity (in both bands) between BL Lacs and FR Is can be accounted for by a single amplification factor, taking into account the scatter of the data, with corresponding jet bulk Lorentz factors $\Gamma \approx 4-6$ (Chiaberge et al. 2000). Similar values in FR I objects have been deduced by Giovannini et al. (2001) from radio data. However these Lorentz factors are significantly smaller than those derived for BL Lacs from other observational and theoretical considerations. Namely, the variability and time lags at different frequencies, the spectral fittings of the overall SED, and the detection of $\gamma$-ray emission constrain $\Gamma \gtrsim 15$ (Dondi \& Ghisellini 1995; Ghisellini et al. 1998; Tavecchio et al. 1998).

Chiaberge et al. (2000) have shown that the presence of a velocity structure in the jet (a fast spine surrounded by a slower layer) could overcome this difficulty. In such a picture, 


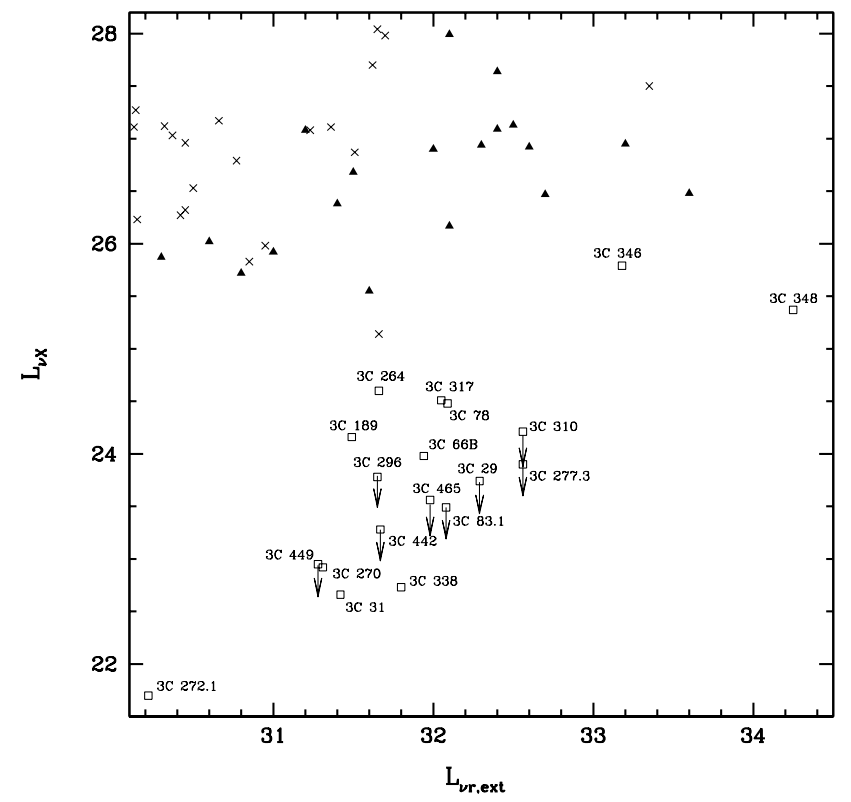

Fig. 2. Core luminosities $L_{v \mathrm{X}}\left(\mathrm{erg} \mathrm{s}^{-1} \mathrm{~Hz}^{-1}\right.$ at $\left.1 \mathrm{keV}\right)$ in the $\mathrm{X}$-ray band of $\mathrm{BL}$ Lacs and radio galaxies (the latter ones indicated by empty squares) vs. the extended radio luminosity $L_{v \mathrm{r}, \mathrm{ext}}\left(\mathrm{erg} \mathrm{s}^{-1} \mathrm{~Hz}^{-1}\right.$ at $1.4 \mathrm{GHz}$.). Filled triangles: LBL; crosses: HBL.

observers at different viewing angles see different components of the jet: the fast spine dominates the emission in BL Lacs, whereas the slower layer dominates the emission observed in FR Is. We remark that such a model has been suggested, from radio observation, for $3 \mathrm{C} 31$ (even though on a much larger scale; Laing \& Bridle 2002).

Here we adopt the same strategy for the X-ray nuclear luminosities. In Fig. 2 we show our samples of FR Is and BL Lacs in the plane formed by $L_{v \mathrm{X}}$ against $L_{v \text { r,ext }}$ (i.e. the nuclear X-ray luminosity vs the extended radio luminosity at $1.4 \mathrm{GHz}$ ). Note that the two classes of BL Lacs share the same range of X-ray luminosity independently on $L_{v \mathrm{r}, \mathrm{ext}}\left(L_{v \mathrm{X}} \sim 10^{27} \mathrm{erg} \mathrm{s}^{-1} \mathrm{~Hz}^{-1}\right.$, with a scatter of $\sim \pm 1$ order of magnitude).

The presence of several upper limits in the FR Is makes the estimate of the relative beaming factor in the X-ray band less accurate than in the radio and optical bands. Although a correlation between $L_{v \mathrm{X}}$ and $L_{v \mathrm{r} \text {,ext }}$ in the FR Is cannot be firmly established, we followed the method used in Capetti et al. (2001) and Chiaberge et al. (2000), and fitted a regression line to the data, using the survival analysis package ASURV (Isobe \& Feigelson 1985, 1986). In this way, we derived that at $L_{v \mathrm{r} \text {,ext }} \sim 10^{32} \mathrm{erg} \mathrm{s}^{-1} \mathrm{~Hz}^{-1}$, which is the median value for our sample, the corresponding X-ray luminosity is $L_{v \mathrm{X}} \sim 10^{23.5} \mathrm{erg} \mathrm{s}^{-1} \mathrm{~Hz}^{-1}$. Thus the luminosity ratio between FR Is and BL Lacs is of $\sim 3.5$ orders of magnitude. This corresponds to a ratio between the Doppler factors of BL Lacs and FR Is $R_{\delta} \equiv \delta_{\mathrm{BL}} / \delta_{\mathrm{FR} \mathrm{I}}=\left(L_{v \mathrm{X}}^{\mathrm{BL}} / L_{v \mathrm{X}}^{\mathrm{FR} \mathrm{I}}\right)^{1 /(p+\alpha)}$ between 10 and 25 for $\alpha_{\mathrm{X}}=1.5-0.5$, respectively, and $p=2$ (as appropriate for a continuous jet $)^{2}$. In the case of a relativistically moving sphere ( $p=3$ ) is $R_{\delta} \sim 6-10$, for the same range of $\alpha_{\mathrm{X}}$. These values

\footnotetext{
2 This relation between the luminosities and the Doppler factors strictly holds for synchrotron and Self-Synchro-Compton (SSC) emissions, while for External Compton emission (EC) we should shift
}

are therefore compatible with those found for both the radio and the optical bands $\left(R_{\delta} \sim 18-20\right.$ for $p=2 ; R_{\delta} \sim 7-9$ for $p=3$ ), although they have a larger allowed range, due to the uncertainty on the local X-ray spectral index.

If there is a velocity gradient across the jet, $R_{\delta}$ corresponds to an "effective" beaming factor, which accounts for the effects of the different jet speed components. Therefore, the comparison of the nuclear luminosity of BL Lacs and FR Is does not lead to a "real" value of a unique Lorentz factor of the jet. As in the case of the radio and optical bands, the inferred values of the bulk Lorentz factors are lower than those obtained for the BL Lacs from other arguments. Indeed, by adopting the reference value of $\delta_{\mathrm{BL}} \approx \Gamma=15 \div 20$ (see Chiaberge et al. 2000, and references therein) and a viewing angle for BL Lacs and FR Is of $\theta=1 / \Gamma$ and $\theta \sim 60^{\circ}$ respectively, we obtain $R_{\delta} \sim 100 \div 200$. This value of $R_{\delta}$ would translate in a ratio $L_{v \mathrm{X}}^{\mathrm{BL}} / L_{v \mathrm{X}}^{\mathrm{FR} \mathrm{I}}=10^{5}-10^{9}$ for the range of parameters used above $\left(p=2,3 ; \alpha_{X}=0.5,1.5\right)$. Therefore, we outline that also the X-ray data are consistent with the possible presence of a velocity structure in the jet.

A second deduction that can be made from the $L_{v \mathrm{r}, \text { ext }} / L_{v \mathrm{X}}$ plane is an estimate of $R_{\delta}$ for each individual source comparing its X-ray luminosity with BL Lacs of similar extended radio luminosity. However this comparison is meaningful only if the difference in X-ray luminosity at a given extended luminosity can be ascribed mainly to a different orientation of the radio galaxy. As already noted in the optical and radio bands, it is also the case in the X-rays that we have a clear indication that objects seen at a small angle with respect to the line of sight are over-luminous. In particular, all sources with a resolved optical jet, an indication of a close jet alignment (see Sparks et al. 1995, 2000), namely 3C 66B, 3C 78, 3C 189, 3C 264 and 3C 346, are among the brightest objects. At the same time we note the low luminosity of 3C 338 $\left(L_{\mathrm{BL}} / L_{\mathrm{FR} \mathrm{I}} \approx 10^{4.5 \pm 1}\right)$ which suggests a largely de-beamed nuclear emission, consistent with a jet basically projected on the sky plane, as deduced from radio data $\left(\theta \approx 85^{\circ}\right.$, Giovannini et al. 2001). The spread in X-ray luminosity then appears to be dominated by orientation effects rather than by intrinsic differences from source to source and therefore this information can be used to constraint the "beaming tracks" that will be discussed in the following section.

Finally, we note that on average HBLs are characterized by lower extended radio luminosities than LBL: the former sample extends from $\sim 10^{29}$ to $10^{31.5} \mathrm{erg} \mathrm{s}^{-1} \mathrm{~Hz}^{-1}$, the latter from $\sim 10^{30} \mathrm{erg} \mathrm{s}^{-1} \mathrm{~Hz}^{-1}$ to $10^{33} \mathrm{erg} \mathrm{s}^{-1} \mathrm{~Hz}^{-1}$ (Chiaberge et al. 2000, and references therein). As the SED's of these two BL Lac classes are different (Fossati et al. 1998), it would be interesting to test if such a distinction also reflects to the FR Is. We might also expect that radio-galaxies of low extended luminosity will show a SED typical of HBL, while the

$1 /(p+\alpha) \rightarrow 1 /(p+1+2 \alpha)$ (Georganopoulos et al. 2001). In such regime of Compton emission it is $0.5 \geq \alpha_{\mathrm{X}} \geq 1$, and for ratios of luminosities of $10^{3.5}$ we overestimate $R_{\delta}$ by a factor $\sim 3.0$ if the emission mechanism is EC. This in principle affects the beaming tracks of flat $\mathrm{X}$-ray spectrum sources but does not critically modify our conclusions considering the scatter of the data in Fig. 2 ( one order of magnitude). 


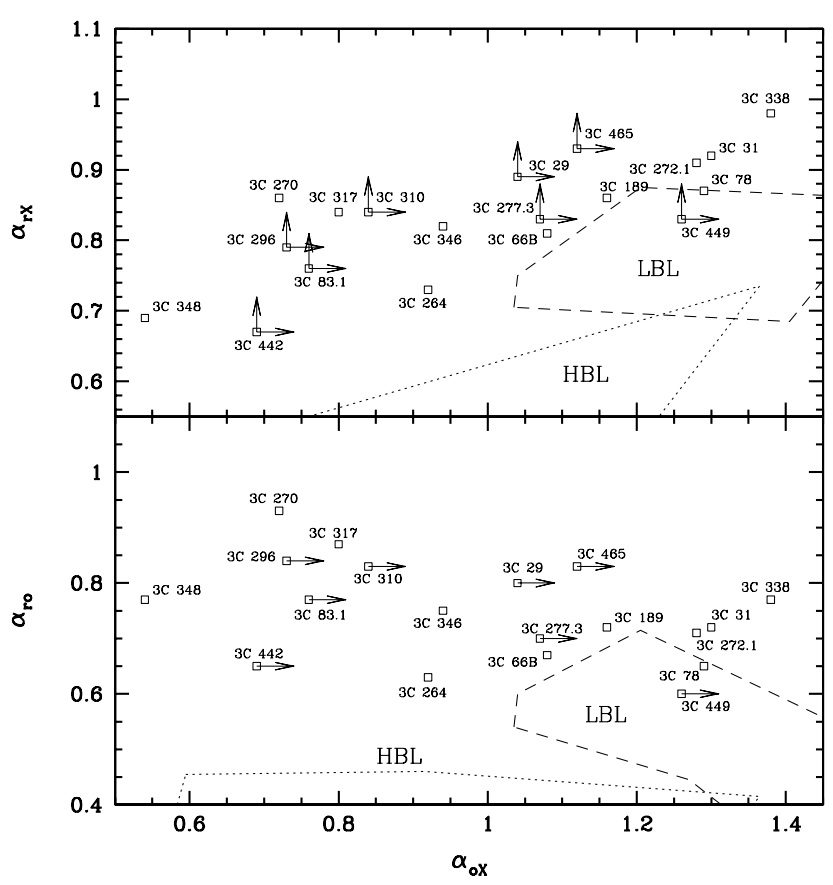

Fig. 3. Plot of the broad-band spectral indices $\alpha_{\mathrm{ro}}$ and $\alpha_{\mathrm{rX}}$ vs. $\alpha_{\mathrm{oX}}$ for our sample, including also the sources of Paper I (3C 288 is not included as it is found $\left.\alpha_{\mathrm{oX}}>0.34\right)$. The regions occupied by LBL and HBL are from Fossati et al. (1998).

highest luminosity objects should be LBL-like. Unfortunately, our FR I sample is mostly concentrated on the transition region across LBL and HBL. The few objects with extreme luminosities and well defined SEDs are 3C 272.1, with $L_{v r \text {,ext }}$ typical of HBL, and the powerful 3C 346 and 3C 348, with the same extended luminosity as LBL. Although clearly not conclusive, these results do not contradict the trend observed in BL Lacs.

\subsection{Distribution of $\alpha_{r o}, \alpha_{r X}$ and $\alpha_{o X}$}

Further information on the unification model can be obtained by considering the broad-band spectral indices $\alpha_{\mathrm{ro}}, \alpha_{\mathrm{rX}}$ and $\alpha_{\mathrm{oX}}$ of FR I radio galaxies and comparing with those of BL Lacs (Fig. 3; the 3CR sources of Paper I are also included).

It is evident that there is essentially no overlap of the distribution of the spectral indices of FR Is with the regions of these planes where HBL and LBL are located. In particular, with respect to HBL a large radio excess is present in radio galaxies (both $\alpha_{\mathrm{ro}}^{\mathrm{FR} \mathrm{I}}$ and $\alpha_{\mathrm{rX}}^{\mathrm{FR} \mathrm{I}}$ are greater than $\alpha_{\mathrm{ro}}^{\mathrm{HBL}}$ and $\alpha_{\mathrm{rX}}^{\mathrm{HBL}}$ respectively). Overall the values of $\alpha_{\mathrm{rX}}$ are similar to those of LBL, but only few sources fall in the LBL region as in most of the FR I objects we have $\alpha_{\mathrm{oX}}<1.1$ and $\alpha_{\mathrm{ro}}>0.7$, i.e. they show an excess of X-ray and radio emission with respect to LBL.

To establish whether the distributions shown in Fig. 3 are indicative of an intrinsic difference between the two classes of objects it is essential to evaluate the effect of the relativistic beaming. This point will be discussed in the following section.

\section{Testing the FR I-BL Lac unified model}

Based on the results outlined in the previous section, it is now possible to perform a more quantitative comparison between FR Is and BL Lacs. In particular, we can test if the information on the SED, spectral indices and difference in luminosities of the two classes can be simultaneously accounted for within the simplest unification scheme, only allowing for the presence of a velocity structure in the jet that is suggested by the observations (see Chiaberge et al. 2000; Capetti et al. 2001).

We reiterate that from the luminosity ratio between a FR Is and BL Lacs in a given band (see Sect. 3.2) we can estimate the ratio of the beaming factors $R_{\delta}$. At the same time, moving from a large viewing angle to a jet more closely aligned with the line of sight the SED is Doppler shifted to higher frequencies by a factor $\propto R_{\delta}$. As the SED is not represented by a single power law, the broad-band spectral indices transform as (Chiaberge et al. 2000):

$\alpha_{i j}^{\mathrm{BL}}=\alpha_{i j}^{\mathrm{FR} \mathrm{I}}+\left(\alpha_{i}-\alpha_{j}\right) \frac{\log R_{\delta}}{\log \left(v_{j} / v_{i}\right)}$

where $i, j \equiv \mathrm{r}, \mathrm{o}, \mathrm{X}$. The above relation provides a "beaming track", i.e. the path of a representative radio galaxy in the $\alpha_{\mathrm{ro}}$ and $\alpha_{\mathrm{rX}}$ vs. $\alpha_{\mathrm{oX}}$ planes as a function of $R_{\delta}$.

It is clear that a crucial ingredient to perform this comparison effectively is the $\mathrm{X}$-ray spectrum of the radio-galaxies. This information is available for eight of our radio galaxies (six from Table 2 and 3C 264 and 3C 270 from Paper I). For the radio emission we have assumed a spectral index $\alpha_{\mathrm{r}}=0$. For the optical band only for few sources in the sample the photometry is sufficiently accurate to allow a constrained enough estimate of $\alpha_{\text {o }}(\approx 0.7-1.3$, see CC99). To be conservative we have assumed the spectral index free to vary in the interval $0.5 \leq \alpha_{\mathrm{o}} \leq 1.5$ (from their standard SED we expect $\alpha_{\mathrm{o}}>1$ and $\alpha_{\mathrm{o}} \leq 1$ for LBL and HBL, respectively).

We then proceed to analyze the tracks for those sources with the best data, by estimating $R_{\delta_{\mathrm{X}}}$ from the ratio between their measured X-ray luminosity and the average luminosity of BL Lac objects (see Fig. 2) and taking into account the $\mathrm{X}$-ray spectral index. Referring in particular to 3C 272.1, which has the best sampled SED, we find $L_{\mathrm{BL}} / L_{\mathrm{FR} \mathrm{I}}=10^{5 \pm 1}$ and $\alpha_{\mathrm{X}}=1.3$, which yields $R_{\delta_{\mathrm{X}}} \approx 10^{1.5 \pm 0.3}$. With this allowed range of values for the ratio of the beaming factors estimated in the $\mathrm{X}$-rays, it is possible to check if, by adopting the same range for the optical and radio band (i.e. $R_{\delta}=R_{\delta_{\mathrm{X}}}$ ), the resulting relative luminosities are also consistent with a given class of BL Lac objects. The tracks of 3C 272.1 fall in the region of overlapping between HBL and LBL (see the shadowed regions in Fig. 4, right panels). Thus the "beamed" 3C 272.1 falls, in both planes, in the HBL zone almost independently of the slope of the optical spectrum (Fig. 4, left panels). We have already seen that the X-ray spectrum of 3C 272.1 is rather steep and its SED has a minimum around the X-ray band, both features typical of HBL. Finally, its extended radio emission is also typical of HBL sources. In summary there is a complete consistency between all spectral and luminosity indicators, and any optical extinction does not modify these conclusions. 


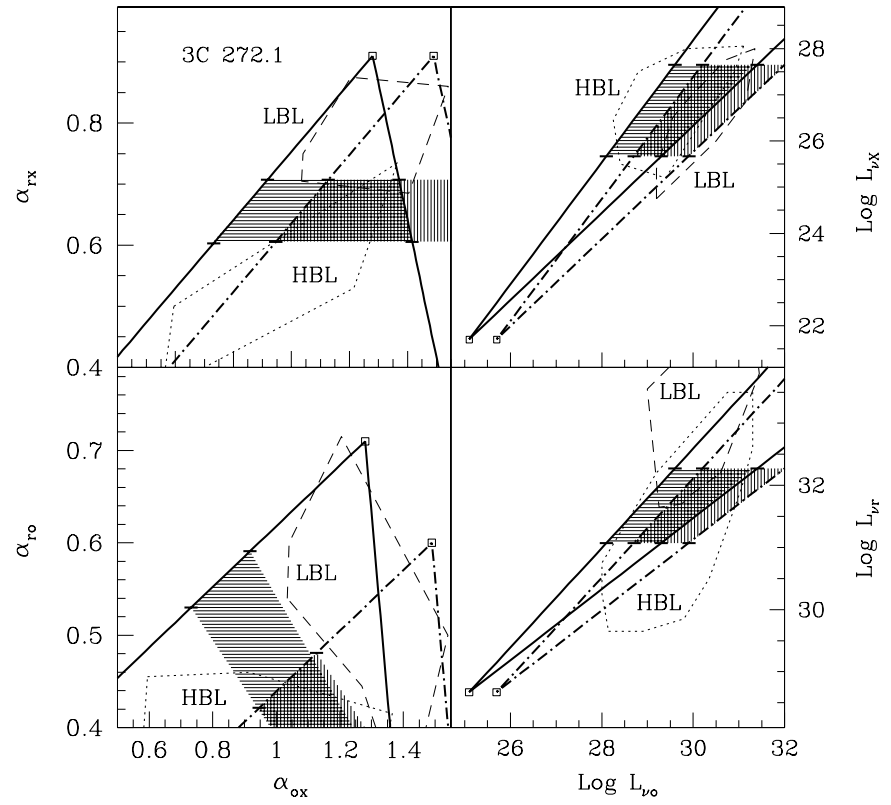

Fig. 4. Left panels: Beaming tracks of $\alpha_{\mathrm{ro}}$ and $\alpha_{\mathrm{rX}}$ vs. $\alpha_{\mathrm{oX}}$ for 3C 272.1 for no absorption (solid lines) and 1.5 mag of extinction (dash-dotted lines) at optical wavelengths. It has been assumed for the spectral indices $\alpha_{\mathrm{X}}=1.3, \alpha_{\mathrm{r}}=0$, and $\alpha_{\mathrm{o}}=0.5$ (leftwards tracks) and $\alpha_{\mathrm{o}}=1.5$ (rightwards tracks). The shadowed regions limit the values of allowed $R_{\delta}$ deduced from the comparison of $L_{\nu \mathrm{X}}$ with $L_{v \mathrm{r} \text { ext }}$ (see Sect. 4). Right panels: Tracks of $L_{v \mathrm{r}}$ and $L_{v \mathrm{X}}$ vs. $L_{v \mathrm{o}}\left(\mathrm{erg} \mathrm{s}^{-1} \mathrm{~Hz}^{-1}\right)$ for the same parameters (upper tracks: $\alpha_{\mathrm{o}}=0.5$, lower tracks: $\alpha_{\mathrm{o}}=1.5$ ).

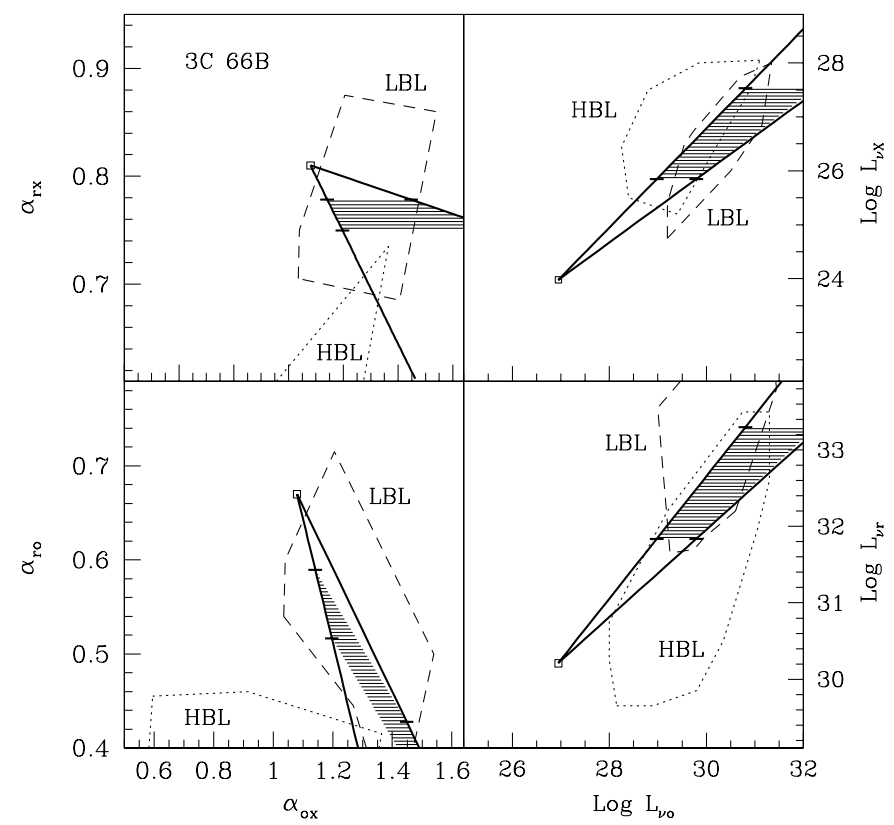

Fig. 5. The same as Fig. 4 for $3 \mathrm{C} 66 \mathrm{~B}$, assuming $\alpha_{\mathrm{X}}=0.3$.

The tracks of $3 \mathrm{C} 66 \mathrm{~B}\left(R_{\delta} \approx 10^{1.4 \pm 0.4}, \alpha_{\mathrm{X}}=0.3\right.$; Fig. 5) "join" the LBL region, as expected by its flat X-ray spectrum and by the shape of its SED, again independently of the optical spectrum. A similar trend holds for 3C 346 for which a small amount of beaming is required due to its high luminosity $\left(R_{\delta} \approx 10^{0.4 \pm 0.4}\right.$; Fig. 6). The X-ray spectral slope $\left(\alpha_{\mathrm{X}}=0.7\right)$ and the extended radio luminosity are typical of LBL. Indeed

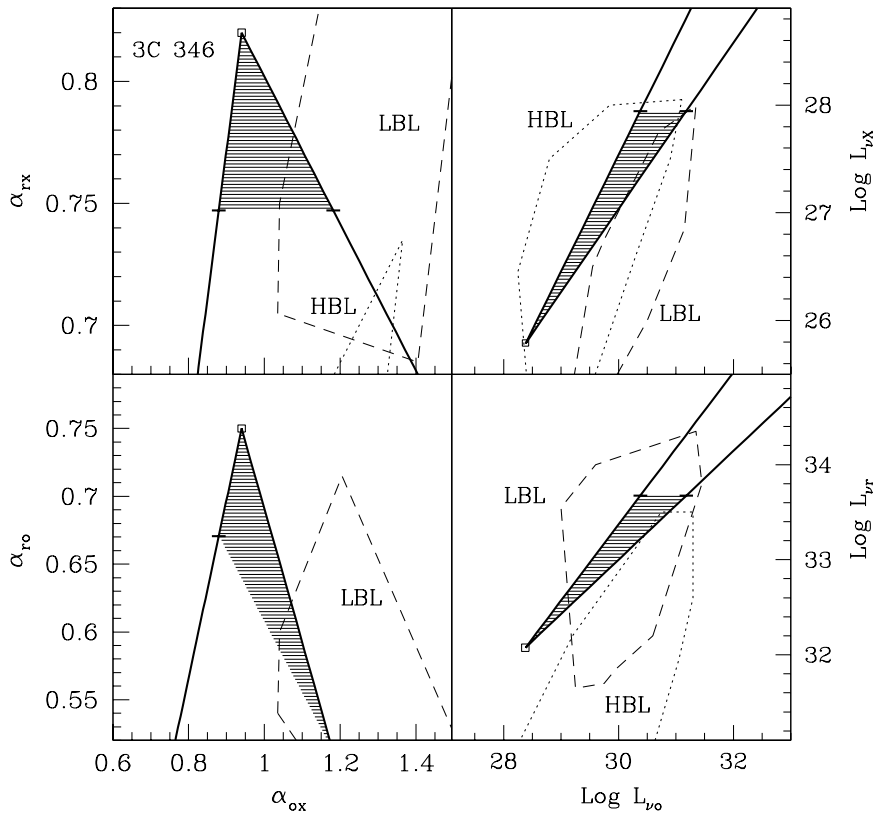

Fig. 6. The same as Fig. 4 for 3 C 346, assuming $\alpha_{X}=0.7$.

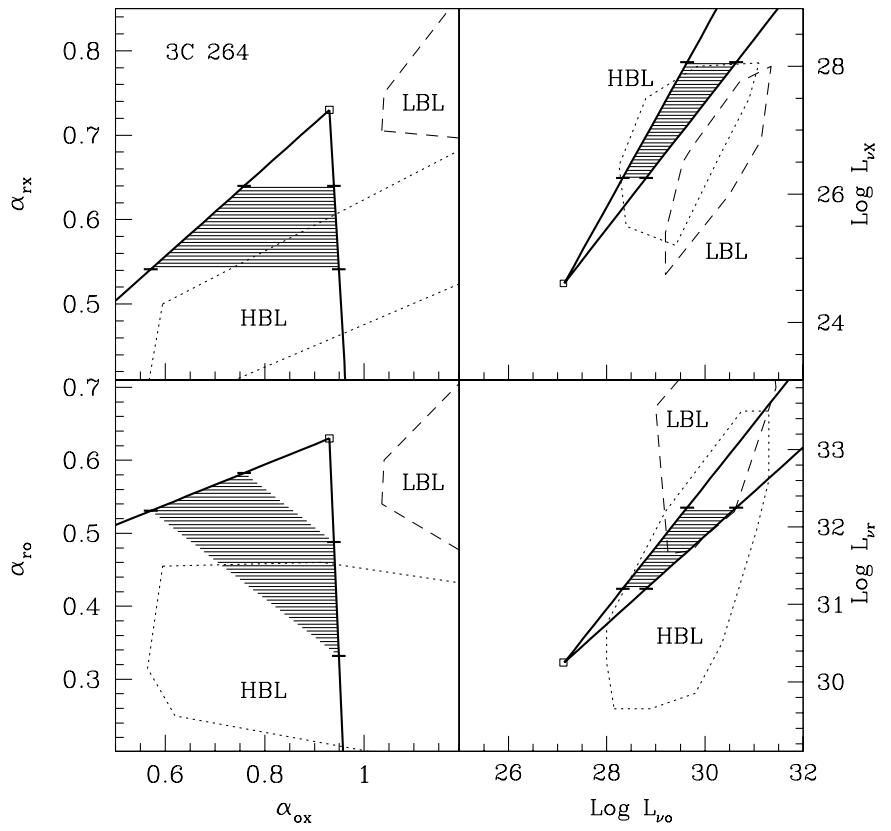

Fig. 7. The same as Fig. 4 for 3C 264, assuming $\alpha_{\mathrm{X}}=1.45$.

3C 346 appears as a LBL-like radio galaxy in all diagnostic planes, provided the optical spectrum is quite steep $\left(\alpha_{\mathrm{o}} \approx 1.5\right)$, consistently with the standard SED of LBL.

Referring to the sources studied in Paper I, the analysis of the beaming tracks confirm that $3 \mathrm{C} 270\left(R_{\delta} \approx 10^{1.5 \pm 0.4}, \alpha_{\mathrm{X}}=\right.$ $0.7)$, can be associated to a LBL, as we already argued based on its SED. As in the case of 3C 346, 3C 270 falls into the LBL region only if its optical spectrum is quite steep $\left(\alpha_{\mathrm{o}} \approx 1.5\right)$ as indeed observed for this source. For $3 C 264\left(R_{\delta} \approx 10^{0.7 \pm 0.3}\right)$ we have an accurate measurement of $\alpha_{\mathrm{X}}(=1.45)$, while from the IR and optical data we can estimate $\alpha_{\mathrm{o}} \approx 0.8-1$. The diagnostic plots (Fig. 7) confirm that this radio galaxy can be associated with a HBL. 
More ambiguous is the case of $3 \mathrm{C} 189\left(R_{\delta} \approx 10^{1 \pm 0.3}\right.$, not shown here $)$ as neither its X-ray spectral slope $\left(\alpha_{\mathrm{X}}=1.1_{-0.4}^{+0.1}\right)$ nor its SED clearly identify it with a given class of BL Lacs. The beaming tracks of the spectral indices seem to favour an association with a LBL, but the available data are not conclusive.

The quality of spectral data on the remaining sources of our sample do not allow us to explore their beaming tracks.

We must remind finally that in Eq. (1) the spectral slopes in the different bands are kept constant, which is in principle inappropriate taking into account that $R_{\delta} \approx 10 \div 30$. This assumption may be critical only in the region of the optical peak and of the transition between the Compton and synchrotron emission. In such a case the values of $\alpha_{\text {ro }}$ and $\alpha_{\text {rX }}$ could be misestimated by a quantity $\Delta \alpha_{i j} \lesssim 0.1$. However we have seen above that a similar amount of uncertainties affect the evaluations of $R_{\delta}$ from Fig. 2.

\section{Summary and conclusions}

We combined measurements in the X-ray band with optical nuclear luminosities derived from HST images and radio data taken from the literature, to characterize the multi-wavelength properties of $20 \mathrm{FR}$ I radio galaxies from the $3 \mathrm{CR}$ sample. In the framework of the unifying model for FR I and BL Lac the results of the present analysis can be summarized as follows:

1. The SED of the sources are not monotonic showing, as in BL Lacs, peaks and minima of emission; in more than half of the objects this minimum occurs around the soft X-ray band.

2. A comparison of the X-ray luminosities of FR Is and BL Lacs indicates that a single amplification factor accounts for their difference in luminosity in the radio, optical and X-ray band.

3. The dominant role of Doppler boosting is confirmed, while obscuration of the nucleus does not appear to be relevant in the FR I/BL Lac unification.

4. The distribution of FR Is in the broad-band spectral index planes shows essentially no overlap with the region typical of HBL and LBL. However, we have shown that this behaviour is only apparent and related to the effects of relativistic beaming on the BL Lac SED.

5. Based on these results we have performed, for the sources with the best data, a more complete and quantitative comparison between FR Is and BL Lacs to verify the consistency between all spectral and luminosity indicators. Namely we derived the beaming factor for 6 FR I sources (including also two objects analyzed in Paper I) based on their X-ray luminosity as compared to that of a BL Lac of similar extended radio luminosity. From the estimate of this single free parameter we predicted optical and radio luminosities and broad-band spectral indices for its counterpart observed at a small angle from the line of sight. This provided us with an association of each FR I source with a given type of BL Lac, which is found to be in agreement with the slope of the X-ray spectrum, the overall SED shape and the extended radio luminosity of the corresponding BL Lac class.

Thus the differences between FR Is and BL Lacs can be all accounted for within the simplest unification scheme, where the nuclear FR I emission is non-thermal radiation from a relativistic jet, only allowing for the presence of a velocity structure in the jet. This is required for Centaurus A, for which very detailed multi-band data up to $\mathrm{GeV}$ energies are available and the orientation of the jet is known (Chiaberge et al. 2001). On the other hand the fact that a similar amount of beaming occurs from radio to X-ray energies puts some strong constraints on the jet dynamics. As it is likely that the X-ray and optical emission regions occur closer to the central engine than the radio emission by $\approx 2$ orders of magnitudes, the jet structure would have to be preserved on these length scales.

In conclusion let us briefly consider how the validity of this procedure to test the unified model could improve with better observational information. Apart the availability of a larger sample of objects, multi-frequency observations would be necessary to estimate the spectral slope at IR-optical-UV wavelengths. Furthermore with respect to previous X-ray data, Chandra is greatly improving our ability to find point-like nuclei. However, in general, they are so weak that only in few cases we had a satisfactory estimate of $\alpha_{\mathrm{X}}$ and $N_{\mathrm{H} \text {,loc }}$ (for this purpose also data from XMM could be useful). Finally we point out that the variability of the core fluxes at all frequencies may affect the shape of the SED and the evaluation of the indices $\alpha_{i j}$ (this may be quite crucial at X-ray energies, see e.g. the case of $3 \mathrm{C} 66 \mathrm{~B})$. A useful step forward to test the validity of the unified scheme would be simultaneous multi-frequency observations.

Acknowledgements. This work has been partially supported by the Italian Space Agency (ASI) and the Italian Ministery for Education and Research (MIUR, cofin 2001/028773). The authors wish to thank M. Georganopoulos for his useful comments and suggestions.

\section{Appendix A: Notes on the X-ray properties of some sources}

We summarize here the observations and the main results of 11 radio galaxies observed with Rosat/PSPC, Chandra, BeppoSAX and ASCA. The properties of the other six sources of the sample are discussed in detail in HW99.

For the analysis of the Rosat/PSPC observations of 3C 29, 3C 66B, 3C 277.3, 3C 189 and 3C 317 (not yet analyzed elsewhere) we have followed the same procedure discussed in Paper I, i.e. we performed a spectral analysis using composite models allowing for the presence of both a thermal and a non-thermal (power-law) component. To establish the statistical significance of the non-thermal component, we have then checked through an F-test when its inclusion improves the fit. The size of the region of photon extraction has been chosen to avoid as much as possible contamination from the extended diffuse zone, and taking into account the intensity of the sources and their position in the detector FOV. In addition for targets offset more than $20^{\prime}$ from the center of the FOV we have excluded the first 50 channels of energy, where the background flux largely increases. The data have been processed using the Exsas package (version 1998) and the reported errors are given at $1 \sigma$ level for a single parameter $\left(\Delta \chi^{2}=1\right)$. The uncertainties on the luminosities (in the range $0.1-2.4 \mathrm{keV}$ ) are at the same 
level, but for two parameters $\left(\Delta \chi^{2}=2.3\right)$. The upper limits are given at $3 \sigma$ levels, while no error is reported for sources where thermal contamination is probable (for the details concerning the statistical criteria for the other sources listed in Table 2, see the quoted literature).

3C 29. X-ray emission from this radio galaxy (observed in 1992 with an exposure of $15197 \mathrm{~s}$ ), offset by $\approx 30^{\prime}$ in the FOV (centered on the cluster A 119), has been detected with a $\approx 3 \sigma$ confidence level (c. $\mathrm{r}$. $=5 \pm 2 \times 10^{-3} \mathrm{~s}^{-1}$ ). Assuming a power law spectrum with $\alpha_{\mathrm{X}}=1.0$ we deduce an unabsorbed flux of $1.0 \times 10^{-13} \mathrm{erg} \mathrm{cm}^{-2} \mathrm{~s}^{-1}$. The X-ray emission is consistent with that expected from the correlation between the radio and $\mathrm{X}$-ray core luminosities (Canosa et al. 1999). To account for a possible contribution from a thermal hot corona then, as for the data of HW99, this emission is assumed as an upper limit to the core luminosity.

3C 31. In Paper I we derived, from Rosat/PSPC data, a nuclear flux of $\approx 2.0 \times 10^{-13} \mathrm{erg} \mathrm{cm}^{-2} \mathrm{~s}^{-1}$, uncertain within a factor $\approx 2(1 \sigma)$ and without any estimate of the spectral index. From the Chandra observation of November 2000 (Hardcastle et al. 2002) the inner region has been resolved in the pointlike nucleus and a jet, with comparable luminosities in the Rosat $\mathrm{X}$-ray band. The spectrum is quite flat $\left(\alpha_{\mathrm{X}}=0.5_{-0.2}^{+0.3}\right)$, with no evidence of a local absorption $\left(N_{\mathrm{H}}=1.5_{-1.4}^{+2.2} \times 10^{21} \mathrm{~cm}^{-2}\right)$. The shape of the SED is very similar to that of $3 \mathrm{C} 189$ (see Fig. 1). The flux is $\approx 5 \times 10^{-14} \mathrm{erg} \mathrm{cm}^{-2} \mathrm{~s}^{-1}$, i.e. $\approx 4$ times less than detected in Rosat but still consistent considering the statistical fluctuations and the jet contribution.

3C 66B. A Chandra observation of November 2000 has shown that non-thermal flux originates from a point-like nucleus and a jet, which are embedded in a diffuse, thermally emitting plasma. From the spectral analysis, an index $1.03_{-0.05}^{+0.07}$ and a flux $2.5 \times 10^{-13} \mathrm{erg} \mathrm{cm}^{-2} \mathrm{~s}^{-1}$ (Hardcastle et al. 2001) have been obtained for the core emission. This radio galaxy falls in the FOV of two pointed PSPC observations of August 1991 (24654 s) and August 1993 (31497 s): in both cases 3C 66B is offset by $38^{\prime}$ from the center, and at $\approx 6^{\prime}$ from the blazar $3 \mathrm{C} 66 \mathrm{~A}$. To avoid as much as possible the contamination from this nearby powerful source, the target photons have been extracted from a ring with radius of only $2.5^{\prime}$, and those of the background from a coronal ring centered on 3C 66A with inner and outer radii of $\approx 3^{\prime}$ and $\approx 8^{\prime}$, respectively. This implies an underestimate of the total luminosity. To search for the core emission we have performed spectral fits with a three components model, thermal (from the diffuse region) plus two power law spectra (from the jet and the core). Keeping fixed the parameters for the thermal and jet emission as deduced from the Chandra data, and assuming $N_{\mathrm{H}}=N_{\mathrm{H}, \mathrm{gal}}\left(=9.15 \times 10^{20} \mathrm{~cm}^{-2}\right)$ we obtain for the two epochs : $\alpha_{\mathrm{X}}=0.20_{-0.25}^{+0.20}$ and flux $9.5 \times 10^{-13} \mathrm{erg} \mathrm{cm}^{-2} \mathrm{~s}^{-1}(1991)$, and $\alpha_{\mathrm{X}}=0.30_{-0.40}^{+0.30}$ and flux $6.0 \times 10^{-13} \mathrm{erg} \mathrm{cm}^{-2} \mathrm{~s}^{-1}$ (1993). Assuming, for consistency with the Chandra results, the largest allowed values of the spectral indices at $2 \sigma$ level of confidence (two related parameters, $\left.\Delta \chi^{2}=4.6\right), \alpha_{\mathrm{X}}=0.75$ (1991) and $\alpha_{\mathrm{X}}=0.85$ (1993), the fluxes are $1.2 \times 10^{-12} \mathrm{erg} \mathrm{cm}^{-2} \mathrm{~s}^{-1}$ and $8.1 \times 10^{-13} \mathrm{erg} \mathrm{cm}^{-2} \mathrm{~s}^{-1}$ for the former and latter observation, respectively. This comparison of the Chandra and Rosat data indicates that the core X-ray flux of $3 \mathrm{C} 66 \mathrm{~B}$ has decreased by a factor $\sim 4$ in 10 years, with a remarkable softening of the spectrum. For the multiwavelength analysis we used the 1993 PSPC data, closer in time to the HST observations.

3C 78. The spectral analysis of a BeppoSAX observation of this source (January 1997) supports the presence of nonthermal emission, but without the estimate of the spectral index (Trussoni et al. 1999). If we assume $\alpha_{\mathrm{X}}=1.0$ we get a flux of $\approx 1.5 \times 10^{-13} \mathrm{erg} \mathrm{cm}^{-2} \mathrm{~s}^{-1}$, corresponding to a value of the luminosity expected from the radio core emission.

3C 189. From a Chandra observation of April 2000 (Worral et al. 2001) the nuclear emission is fitted with a non-thermal spectrum with $\alpha_{\mathrm{X}}=1.1_{-0.4}^{+0.1}$, with flux $3.6 \times 10^{-13} \mathrm{erg} \mathrm{cm}^{-2} \mathrm{~s}^{-1}$. Approximately the same energy flux is emitted by a jet and from a diffuse thermal halo surrounding the central region within a radius of $10^{\prime \prime}$. To verify for a possible variability we have analyzed the data of a Rosat/PSPC observation of 1991. From a spectral fit, assuming a thermal + non-thermal components, we get a slightly softer spectrum, $\alpha_{\mathrm{X}}=1.4_{-0.2}^{+0.2}$, but still consistent with the Chandra observation. The resulting flux is $6.2 \times 10^{-13} \mathrm{erg} \mathrm{cm}^{-2} \mathrm{~s}^{-1}$; fixing $\alpha_{\mathrm{X}}=1.2$ the allowed range of the flux is $3.0-6.7 \times 10^{-13} \mathrm{erg} \mathrm{cm}^{-2} \mathrm{~s}^{-1}$ at $2 \sigma$ level. Then, if $\mathrm{X}$ ray variability occurred in the nucleus of $3 \mathrm{C} 189$ in the period 1991-2000, its amplitude had to be less than a factor $\sim 1.8$. In the present analysis we have adopted the Chandra results.

3C 272.1. The X-ray emission from the nucleus of M 84 detected by Chandra (May 2000) has been fit with a power law spectrum with $\alpha_{\mathrm{X}}=1.3_{-0.1}^{+0.1}, N_{\mathrm{H}}=2.7_{-0.3}^{+0.3} \times 10^{21} \mathrm{~cm}^{-2}$ and an unabsorbed flux of $1.9 \times 10^{-13} \mathrm{erg} \mathrm{cm}^{-2} \mathrm{~s}^{-1}$ (Finoguenov $\&$ Jones 2001). We remark that the hydrogen column density implies an extinction of $\approx 1.4$ mag at optical wavelengths, consistent with the HST data (CCC99). In the Rosat energy band the core luminosity is $5.0 \times 10^{39} \mathrm{erg} \mathrm{s}^{-1}$, in agreement with the data of HW99.

3C 277.3. No X-ray emission has been detected at the position of this radio galaxy associated with the cluster Coma A. The pointing center was offset by $\approx 45^{\prime}$ in the FOV (pointed in 1991 , c.r. $\lesssim 10^{-3} \mathrm{~s}^{-1}$, exposure of 20345 s). Assuming a power law model with $\alpha_{\mathrm{X}}=1$ this corresponds to an upper limit flux of $\$ 4.2 \times 10^{-14} \mathrm{erg} \mathrm{cm}^{-2} \mathrm{~s}^{-1}$, consistent with the radio/X-ray correlation of Canosa et al. (1999).

3C 317. This radio galaxy is the main member of the cooling flow cluster A 2052: its X-ray properties (from HRI observations) were analyzed by Rizza et al. (2000) who did not find strong evidence of a compact core. The PSPC flux from the inner region (c.r. $=0.23 \pm 0.01 \mathrm{~s}^{-1}$, total exposure of $9240 \mathrm{~s}$ from two pointings of 1992 and 1993) is consistent with the emission from a plasma with $T=1.4_{-0.1}^{+0.2} \mathrm{keV}$ and metallicity $=0.60_{-0.10}^{+0.15}\left(\chi_{\mathrm{r}}^{2}=1.2\right)$, while a simple power law is not acceptable $\left(\chi_{\mathrm{r}}^{2}=3\right)$. If we fix the metallicity to 0.5 a better fit is obtained at $94 \%$ of confidence including a nonthermal component, that appears very flat $\left(\alpha_{X} \approx 0.1\right)$ even though the determination of the slope is very uncertain. The flux results to be $\approx 7.4 \times 10^{-13} \mathrm{erg} \mathrm{cm}^{-2} \mathrm{~s}^{-1}$. This object has been observed in September 2000 with the ACIS-S detector on Chandra, for a total exposure of $36754 \mathrm{~s}$. The core emission has been fit to an unabsorbed power law with $\alpha_{\mathrm{X}}=1.00_{-0.15}^{+0.15}$ and flux $\approx 2.1 \times 10^{-13} \mathrm{erg} \mathrm{cm}^{-2} \mathrm{~s}^{-1}$ in the Rosat energy range (Blanton et al. 2002; no jet-like structure has been detected). 
This emission is $\sim 3.5$ times weaker than deduced in the 1993/93 observations and has a steeper spectrum. However the spectral parameters obtained from the PSPC data are affected by large statistical fluctuations and we have verified that they are consistent with the Chandra results at the $\approx 2 \sigma$ level. Then it is not possible to argue about a variability of the nuclear Xray emission. For the analysis of the SED we have adopted the 1992/1993 observations, closer in time to the HST data.

3C 338. A Chandra observation of the cluster A 2199 (December 1999) has detected a point-like source coinciding with the center of the galaxy NGC 6166 (Di Matteo et al. 2001). The low count rate does not provide a well constrained estimate of the parameters: for a power law model we deduce $\alpha_{\mathrm{X}}=0.54_{-0.54}^{+0.56}$ and flux $1.8 \times 10^{-14} \mathrm{erg} \mathrm{cm}^{-2} \mathrm{~s}^{-1}$ (in the Rosat energy band). However it has been found that the value of the spectral slope rapidly increases with the hydrogen column: for $N_{\mathrm{H}, \text { loc }} \lesssim 2 \times 10^{21} \mathrm{~cm}^{-2}$, Di Matteo et al. obtain $\alpha_{\mathrm{X}} \sim 1.9$ and a flux $\approx 2$ times larger.

3C 346. The observations of Rosat/PSPC (1993) and ASCA (1995) of this radio galaxy have been recently analyzed by Worrall \& Birkinshaw (2001). From the Rosat data it turns out that most of the emission is point-like, unabsorbed (local $N_{\mathrm{H}} \lesssim 2 \times 10^{21} \mathrm{~cm}^{-2}$ ) with $\alpha_{\mathrm{X}}=0.69_{-0.14}^{+0.16}$ and flux $=1.1 \times 10^{-12} \mathrm{erg} \mathrm{cm}^{-2} \mathrm{~s}^{-1}$, while from ASCA (two years later) a similar spectral slope $\left(\alpha_{X}=0.73_{-0.23}^{+0.17}\right)$ is found, but a significant lower flux $\left(7.4 \times 10^{-13} \mathrm{erg} \mathrm{cm}^{-2} \mathrm{~s}^{-1}\right)$. For our analysis we have used the 1995 ASCA data.

3C 348. From ASCA (1998) and Rosat data (PSPC in 1993, HRI in 1996) Siebert et al. (1999) detected non-thermal emission from the core of this radio galaxy with very poor determination of the spectral index $\left(\alpha_{\mathrm{X}}=0.7_{-0.3}^{+0.7}\right)$ and flux $\approx 3 \times 10^{-13} \mathrm{erg} \mathrm{cm}^{-2} \mathrm{~s}^{-1}$. This corresponds to $\sim 10 \%$ of the total extended thermal X-ray emission. These results have been confirmed by an observation with BeppoSAX of March 1997 (Trussoni et al. 2001). It is worth noticing that 3C 348 is extremely bright at high energies: the optical core luminosity is consistent with its radio emission (CCC99), while the $\mathrm{X}$-ray luminosity is $\approx$ ten times higher than expected from the radio/X-ray correlation (Canosa et al. 1999).

\section{References}

Bai, J. M., \& Lee, M. G. 2001, ApJ, 548, 244

Blanton, E. L., Sarazin, C. L., \& McNamara, B. R. 2003, ApJ, 585, 227

Canosa, C. M., Worrall, D. M., Hardcastle, M. J., \& Birkinshaw, M. 1999, MNRAS, 310, 30

Capetti, A., \& Celotti, A. 1999, MNRAS, 304, 434 (CC99)

Capetti, A., Trussoni, E., Celotti, A., Feretti, L., \& Chiaberge, M. 2000, MNRAS, 318, 493 (Paper I)

Capetti, A., Celotti, A., Chiaberge, M., et al. 2001, A\&A, 383, 184

Chiaberge, M., Capetti, A., \& Celotti, A. 1999, A\&A, 349, 77 (CCC99)
Chiaberge, M., Capetti, A., Celotti, A., \& Ghisellini, G. 2000, A\&A, 358,104

Chiaberge, M., Capetti, A., \& Celotti, A. 2001, MNRAS, 324, L33

Chiaberge, M., Macchetto, F. D., Sparks, et al. 2002, ApJ, 571, 247

Di Matteo, T., Quataert, E., Allen, S. W., Narayan, A. C., \& Fabian, A. C. 2000 , MNRAS, 311,507

Di Matteo, T., Johnstone, R. M., Allen, S. W., \& Fabian, A. C. 2001, ApJ, 550, L19

Di Matteo, T., Allen, S. W., Fabian, A. C., Wilson, A. S., \& Young, A. J. 2002, ApJ, 582, 133

Dondi, L., \& Ghisellini, G. 1995, MNRAS, 273, 583

Fabbiano, G. 1989, ARA\&A, 27, 1

Fanaroff, B. L., \& Riley, J. M. 1974, MNRAS, 167, 31p

Finoguenov, A., \& Jones, C. 2001, ApJ, 547, L107

Fossati, G., Maraschi, L., Celotti, A., Comastri, A., \& Ghisellini, G. 1998, MNRAS, 299, 433

Georganopoulos, M., Kirk, J. K., \& Mastichiadis, A. 2001, ApJ, 561, 111

Ghisellini, G., Celotti, A., Fossati, G., Maraschi, L., \& Comastri, A. 1998, MNRAS, 301, 451

Giommi, P., \& Padovani, P. 1995, ApJ, 444, 567

Giovannini, G., Feretti, L., Gregorini, L., \& Parma, P. 1988, A\&A, 199,73

Giovannini, G., Cotton, W. B., Feretti, L., Lara, L., \& Venturi, T. 1998, ApJ, 493, 632

Giovannini, G., Cotton, W. B., Feretti, L., Lara, L., \& Venturi, T. 2001, ApJ, 552, 508

Hardcastle, M. J., Alexander, P., Pooley, G. G., \& Riley, J. M. 1996, MNRAS, 278, 273

Hardcastle, M. J., \& Worrall, D. M. 1999, MNRAS, 309, 969 (HW99)

Hardcastle, M. J., \& Worrall, D. M. 2000, MNRAS, 314, 359

Hardcastle, M. J., Birkinshaw, M., \& Worrall, D. M. 2001, MNRAS, 326, 1499

Hardcastle, M. J., Worrall, D. M., Birkinshaw, M., Laing, R. A., \& Bridle, A. H. 2002, MNRAS, 334, 182

Isobe, T., \& Feigelson, E. 1985, ApJ, 293, 192

Isobe, T., \& Feigelson, E. 1986, ApJ, 306, 490

Laing, R. A., \& Bridle, A. H. 2002, MNRAS, 336, 328

Loewenstein, M., Mushotzky, R. F., Angelini, L., Arnaud, K. A., \& Quataert, E. 2001, ApJ, 555, L21

Morganti, R., Killeen, N. E. B., \& Tadhunter, C. N. 1993, MNRAS, 263,1023

Narayan, R., \& Yi, I. 1995, ApJ, 444, 231

Pellegrini, S., Venturi, T., Comastri, A., et al. 2003, ApJ, 585, 677

Rizza, E., Loken, C., Bliton, M., et al. 2000, AJ, 119, 21

Siebert, J., Kawai, N., \& Brinkmann, W. 1999, A\&A, 350, 25

Sparks, W. B., Golombek, D., Baum, S. A., et al. 1995, ApJ, 450, L55

Sparks, W. B., Baum, S. A., Biretta, J., Macchetto, F. D., \& Martel, A. 2000, ApJ, 542, 667

Tavecchio, F., Maraschi, L., \& Ghisellini, G. 1998, ApJ, 509, 608

Trussoni, E., Vagnetti, F., Massaglia, S., et al. 1999, A\&A, 348, 437

Trussoni, E., Feretti, L., Massaglia, S., \& Parma, P. 2001, A\&A, 366, 788

Urry, C. M., \& Padovani, P. 1995, PASP, 107, 803

Worrall, W. D., \& Birkinshaw, M. 2001, ApJ, 551, 178

Worrall, W. D., Birkinshaw, M., \& Hardcastle, M. J. 2001, MNRAS, 326, L7 